\title{
Quistes intraneurales del nervio ciático poplíteo externo en edad pediátrica: presentación de 2 casos y revisión de la literatura
}

\author{
J. Robla-Costales; M. Socolovsky*,**; A. Dubrovsky**; F. Astorino***; J. Fernández-Fernández; J. Ibáñez-Plágaro \\ y J. García-Cosamalón \\ Hospital de León, España. Hospital de Clínicas*, Universidad de Buenos Aires, Argentina. Instituto de Neurociencias**, Fundación Favaloro, \\ Buenos Aires, Argentina. Hospital de Niños***, Santa Fe, Argentina.
}

\section{Resumen}

Los quistes intraneurales son lesiones benignas de contenido mucinoso que se ubican dentro del epineuro de algunos nervios periféricos. El ciático poplíteo externo es el nervio más afectado por esta entidad, de incidencia muy baja y cuya etiopatogenia ha sido motivo de controversia. Spinner et al. en el año 2003 publican su teoría articular, que explica la formación de estas lesiones y la causa de su recidiva frecuente tras su evacuación quirúrgica. Esta teoría, a día de hoy, es la más aceptada, y desde su aplicación ha demostrado ser importante en la reducción de las recidivas y la mejora de los resultados del tratamiento de los quistes intraneurales. La mayoría de las series de casos publicadas describen las características clínicas, radiológicas y el pronóstico de esta entidad en adultos. A través de la revisión de los casos pediátricos existentes en la literatura, y junto con 2 nuevos casos que presentamos, este trabajo revisa los aspectos clínicos de los quistes intraneurales en su presentación pediátrica y las diferencias con su presentación en adultos. Es importante conocer esta entidad ya que es uno de los diagnósticos diferenciales que deben ser tenidos en cuenta al estudiar un paciente con un pie caído, y su tratamiento correcto en tiempo y forma redunda muchas veces en una remisión completa de la sintomatología.

PALABRAS CLAVE: Quistes intraneurales. Ganglión intraneural. Nervio ciático poplíteo externo. Steppage. Tumor de nervio periférico.

Intraneural cysts of the peroneal nerve in childhood: report of 2 cases and literature review

\section{Summary}

Intraneural cysts are benign lesions filled with mucinous content and located inside the epineurum of the

Recibido: 11-06-10. Aceptado: 26-09-10 peripheral nerves. Peroneal nerve is the most affected nerve. The entity is rare and its ethiopathology still remains to be definitely elucidated. In 2003 Spinner et al published their articular theory, implicated in the formation and frequent recurrence of these lesions after surgical treatment. The practical application of this theory, nowadays almost universally accepted, generated an important diminution in the recurrence rate after surgical evacuation of this lesions, previously very elevated. Most of the cases of this entity are described in adults. In the present paper we describe two pediatric cases and analyze the clinical aspects of these cysts in pediatric and adults patients. Peroneal intraneural cysts are one of the differential diagnoses in foot drop, and an adequate treatment concludes in a complete remission of the symptoms.

KEY WORDS: Intraneural cysts. Intraneural ganglion. Peroneal nerve. Steppage. Peripheral nerve tumor.

\section{Introducción}

Desde su primera descripción en 1810 , realizada por Beauchêne ${ }^{7,8}$, los quistes intraneurales han sido motivo de interés y estudio por parte de numerosos autores. Estas lesiones no neoplásicas se encuentran en el epineuro de los nervios periféricos. El nervio ciático poplíteo externo (CPE), también llamado peroneo, es el más frecuentemente afectado. En dicho nervio, la lesión se presenta a nivel del cuello quirúrgico del peroné, inmediatamente proximal y distal al sitio donde se bifurca en sus dos ramas principales, la superficial y la profunda o tibial anterior. Debido a la baja incidencia de estas lesiones, su etiología sigue siendo un tema controvertido. La teoría más aceptada actualmente es la articular, descrita por Spinner ${ }^{10-17}$, la cual sostiene que el origen verdadero de estas lesiones es a partir de la articulación tibioperonea superior, y que mediante la disección del epineuro de una pequeña rama del nervio ciático

Abreviaturas. CPE: nervio ciático popliteo externo.RM: resonancia magnética. TAC: tomografía axial computarizada. 
poplíteo externo, la rama articular, el contenido mucinoso se dirige en sentido proximal hasta afectar el tronco principal del nervio y sus dos ramas terminales principales. Esta pequeña rama tiene origen justamente en la bifurcación del nervio, siendo entonces ésta en verdad una trifurcación. Los quistes intraneurales son infrecuentes en los adultos, y extremadamente raros en niños. Presentamos dos nuevos casos de pacientes pediátricos de 6 y 9 años de edad y revisamos los aspectos de esta entidad, enfocado en su presentación pediátrica.

\section{Caso 1}

Historia. Niña de 6 años de edad valorada en la consulta por presentar una marcha en Steppage debido a una parálisis del nervio CPE izquierdo. La paciente no presentaba antecedentes médicos de interés ni historia familiar de trastornos neurológicos. La sintomatología había comenzado cuatro meses atrás con déficit motor distal progresivo de la extremidad inferior izquierda, asociado a dolor en la cara lateral de la pierna izquierda y el pie. No hubo antecedentes traumáticos en la extremidad, de acuerdo al interrogatorio realizado a la paciente y su familia. El examen físico reveló una marcha en Steppage, debida a una paresia grave para la flexión dorsal del pie y de los dedos del pie izquierdo, apreciándose además una atrofia leve de la musculatura de la pierna. En la cara lateral de la pierna izquierda, a nivel del cuello del peroné, se apreció a la palpación una masa en el trayecto del nervio peroneo, cuya percusión producía un signo de Tinel positivo. La paciente presentaba una fuerza 0/5 para la flexión dorsal del tobillo, 1/5 para la extensión de los dedos del pie, mientras que la eversión del pie estaba casi completamente conservada $(4 / 5)$.

Los estudios de conducción nerviosa revelaron una latencia prolongada del nervio CPE izquierdo a nivel de la rodilla por encima de la cabeza del peroné. La electromiografía mostró denervación grave de los músculos del compartimento anterior de la pierna izquierda con pérdida de unidades motoras, pero los músculos peroneo largo y corto no presentaron signos de denervación. La latencia al músculo pedio después de estimular el nervio CPE en el cuello del peroné estaba incrementada y se observó una diferencia significativa cuando la estimulación del nervio se realizaba por encima o por debajo de la cabeza del peroné. La resonancia magnética $(\mathrm{RM})$ de la rodilla y la pierna izquierda reveló un nervio CPE aumentado de tamaño debido a una masa quística lobulada (45 mm de largo) a nivel del cuello del peroné. El contenido del quiste era hipointenso en $\mathrm{T} 1$ e hiperintenso en relación con el músculo en T2-spin eco con algunas áreas hipointensas. También se observaron cambios en la señal del músculo en consonancia con la denervación de la musculatura del
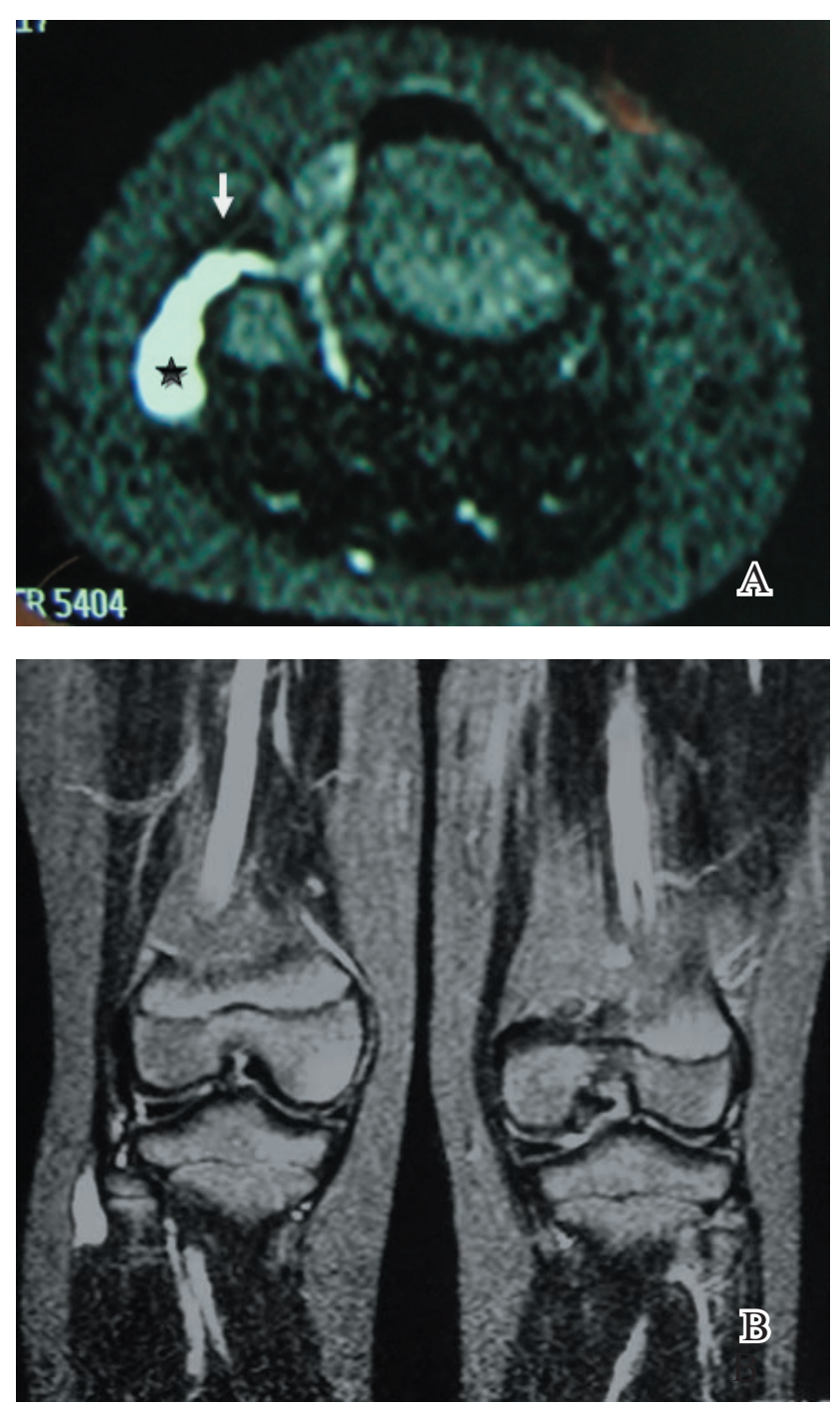

Fig. 1. A. Corte axial de RM de rodilla de Caso 1, demostrando la relación del quiste intraneural (asterisco) con la articulación tibioperonea superior, a través de la rama articular (flecha blanca). B. Corte coronal de RM en la misma paciente, en el que se aprecia el nervio ciático popliteo externo afectado por el quiste intraneural a nivel de la cabeza del peroné.

compartimento anterior y lateral, en comparación con el compartimento posterior de la misma pierna. En los cortes axiales y coronales de la RM en T2 se evidenció la existencia de una conexión entre la masa quística y la articulación tibioperonea superior, el signo de la cola (Fig. 1 A y B).

Intervención quirúrgica. Se realizó un abordaje en "S itálica" centrado en la lesión mediante palpación. Tras la disección de los planos superficiales, se abrió el túnel fibular proximal y se identificaron la lesión quística, el nervio CPE y su trifurcación en las ramas superficial, pro- 


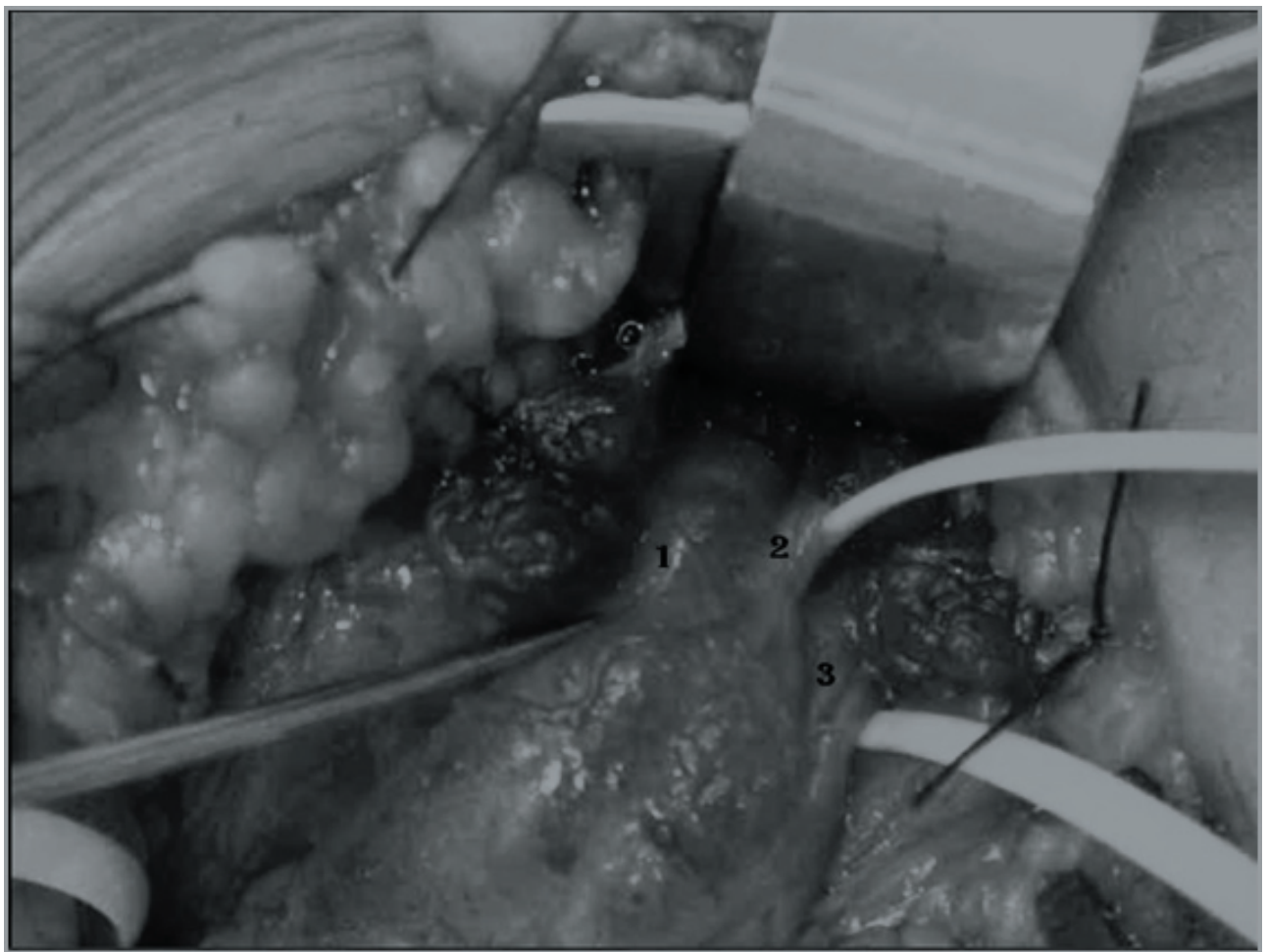

Fig. 2. Exposición del nervio ciático poplíteo externo y su trifurcación en las ramas articular (1), superficial (2) y profunda (3); las tres ramas del nervio se aprecian afectadas por la expansión del quiste intraneural.

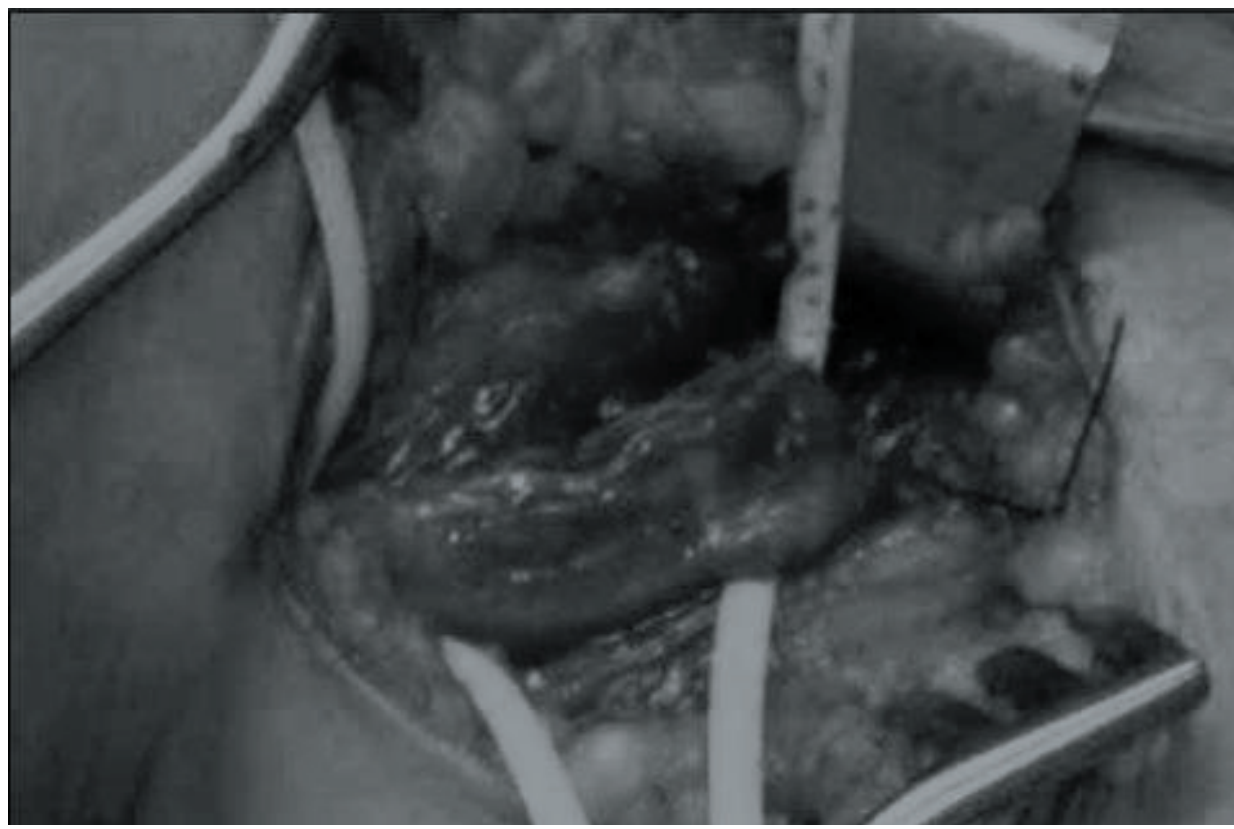

Fig. 3. Fotografía del nervio ciático popliteo externo tras la descompresión del quiste intraneural y posterior ligadura y sección de la rama articular para evitar recidivas posteriores.

funda y articular. Las tres ramas se encontraban afectadas por la expansión del quiste (Fig. 2). La rama articular fue disecada y se expuso en toda su extensión, confirmando el curso del quiste hacia la articulación tibioperonea superior. Después de localizar una zona en la superficie del quiste libre de fascículos nerviosos (mediante visualización directa con magnificación óptica y estimulación intraoperatoria), se descomprimió el quiste a través de 
una incisión longitudinal epineural, liberando el quiste un líquido viscoso claro. La pared del quiste no fue resecada. Por último, la rama articular fue ligada y seccionada para evitar futuras recidivas (Fig. 3). La articulación tibioperonea superior se dejó intacta. El examen anatomopatológico confirmó el diagnóstico de quiste intraneural.

Curso postoperatorio. La RM postoperatoria a los 3 meses no mostró recidiva del quiste. Durante el seguimiento postoperatorio, se constató una recuperación funcional grado $4+$ para la flexión dorsal del tobillo y la extensión de los dedos del pie a los 6 meses y grado 5 - al año de la intervención.

\section{Caso 2}

Historia. Un niño de 9 años de edad comenzó a quejarse de dolor sobre la parte lateral del pie y falta de fuerza en la pierna derecha cuando practicaba deporte. Tres meses después, ya se evidenciaba una clara paresia para la flexión dorsal del pie, por lo que fue evaluado inicialmente por neurología y luego por neurocirugía. El paciente no presentaba antecedente traumático en la extremidad afectada. El examen físico evidenció una marcha en steppage, con una paresia grave para la extensión y la eversión del pie y la extensión de los dedos del pie (grado 1/5). A la palpación, se apreciaba claramente una masa a nivel del cuello del peroné, con un signo de Tinel presente.

Los estudios de conducción nerviosa de la extremidad inferior derecha evidenciaban la denervación aguda y crónica de los músculos inervados por las ramas superficial y profunda del nervio CPE. La amplitud y la latencia del nervio peroneo fueron patológicas a nivel del cuello del peroné.

La resonancia magnética de la rodilla derecha mostró una masa quística a lo largo del trayecto del nervio CPE a nivel del cuello del peroné, con una extensión de $52 \mathrm{~mm}$, partiendo de la parte lateral de la fosa poplítea. Al igual que en el caso 1, el contenido del quiste fue hipointenso en $\mathrm{T} 1$ e hiperintenso en relación con el músculo esquelético en T2, con áreas hipointensas. También se apreciaban cambios relacionados con la denervación de la musculatura del compartimento anterior y lateral. No se evidenció el signo de la cola en las imágenes de RM, pero se sospechó la presencia de un quiste intraneural dada la presencia de una masa no sólida de crecimiento lento en el trayecto del nervio ciático poplíteo externo cerca de su bifurcación.

Intervención quirúrgica. Similar a la paciente 1, en este caso el abordaje permitió identificar las tres ramas del nervio, así como la lesión de contenido quístico. La estimulación intraoperatoria permitió identificar ambos ramos motores. La resección de la lesión fue completa e incluyó la ligadura y posterior sección del pequeño ramo articular.

Curso postoperatorio. La evaluación al año evidenció una recuperación completa del déficit previo, y la RM descartó cualquier signo de recidiva de la lesión.

\section{Discusión}

\section{Consideraciones generales sobre los quistes intraneurales}

Los quistes intraneurales son lesiones quísticas no neoplásicas que se forman en el epineuro de los nervios periféricos. La primera descripción de esta lesión fue realizada por Beauchêne en $1810^{7,8}$.

Estas lesiones pueden aparecer en diferentes nervios, aunque el ciático poplíteo externo a nivel del cuello del peroné es con diferencia la localización más frecuente ${ }^{1,7,12,14}$. En cuanto a la distribución por sexo, estas lesiones se presentan con mayor frecuencia en los hombres que en las mujeres, representando el $80 \%$ de pacientes ${ }^{8}$.

Debido a la rareza de los quistes intraneurales, su etiopatogenia ha sido motivo de controversia durante los últimos dos siglos ${ }^{12,16}$. Durante años, el manejo de estas lesiones se ha basado en la experiencia de casos aislados publicados, en lugar de la comprensión del mecanismo de formación de quistes. Las tasas de recurrencia publicadas han sido hasta del $30 \%^{1,3,4,12,14,16}$, e inclusive podrían ser aún mayores ya que, en muchos casos publicados, no se realizaron estudios de imágenes postoperatorios con RM. Actualmente, el reconocimiento de la teoría articular formulada por Spinner et al. en el año $2003^{12}$, ha demostrado ser importante en la reducción de las recidivas y la mejora de los resultados del tratamiento de los quistes intraneura$\operatorname{les}^{3,5,6,12,13,14}$. Esta teoría, fundamentada sobre datos clínicos, radiológicos, anatómicos y exámenes histológicos, explica la patogénesis y el patrón de propagación de los quistes intraneurales del nervio CPE. Otros artículos publicados por Spinner et al. han aportado más datos para justificar esta teoría ${ }^{2,12-17}$. Los quistes intraneurales del nervio CPE están en continuidad directa con la cápsula articular de la articulación tibioperonea superior. Esta conexión se establece a través de la rama articular del nervio CPE, y se ha demostrado un mecanismo valvular unidireccional desde la articulación hacia la rama articular. Desgarros en la cápsula articular, producidos o favorecidos por micro o macro-trauma así como por cambios degenerativos en la articulación tibioperonea superior, permiten el paso del líquido sinovial desde la articulación al espacio epineural. Este espacio, virtual en individuos sanos, es disecado por el contenido mucinoso del quiste. Con la elevación de la presión intraarticular, se puede producir la propagación del quiste, disecando el epineuro del nervio a través de las zonas de menor resistencia ${ }^{12,16}$.

Los quistes intraneurales del nervio CPE suelen presen- 
tarse en hombres adultos, con una clínica típica de dolor en la rodilla o en la región proximal de la pierna previo a la aparición de debilidad o alteraciones sensitivas en la distribución del nervio CPE. El antecedente de traumatismo previo en la rodilla y la presencia de una masa palpable en la región externa de la rodilla son comunes. La artrosis se encuentra frecuentemente asociada. El signo de Tinel puede ser positivo a la palpación de la región externa de la rodilla. Suele producirse de forma gradual un déficit motor en forma de "pie caído", por una debilidad variable de los músculos tibial anterior, peroneos largo y corto y extensores de los dedos $1,3,4,5,9,18$. La afectación motora es siempre mayor que la sensitiva, la cual es infrecuente y por lo general leve ${ }^{8,9}$. Típicamente los pacientes presentan una lesión predominantemente de la rama profunda del nervio CPE, aunque los pacientes pueden presentar una afectación mayor del nervio CPE, incluso en algunos casos se puede encontrar también afectación de la división tibial del nervio ciático común. Se ha postulado que la presencia de dolor en la rodilla y el diagnóstico clínico de una lesión de la rama profunda del CPE, especialmente si se relaciona con una masa quística en la región lateral de la rodilla, son patognomónicos de esta entidad ${ }^{10,12}$.

El examen físico del paciente incluye la palpación de la región externa de la rodilla para valorar una posible tumoración, y la evaluación de la función motora y sensitiva para confirmar posibles déficits neurológicos del nervio ciático o de sus divisiones tibial y peronea. Estos hallazgos en la exploración pueden ser confirmados mediante electromiografía y estudios de conducción nerviosa. Estos estudios son muy útiles para determinar la extensión y magnitud de la lesión. La electromiografía puede demostrar una degeneración completa del nervio sin respuesta a la estimulación ${ }^{1,4,18}$. Los estudios de imagen (TAC, ecografía, y sobre todo la RM) pueden ayudar a establecer el diagnóstico preoperatorio. La RM es capaz de demostrar la naturaleza quística de la lesión, siendo hipointensa y homogénea en T1 e hiperintensa en T2. La alta resolución de la RM permite demostrar conexiones del quiste intraneural con la articulación ${ }^{1,4,6,10,13,16}$.

El diagnóstico diferencial de las lesiones quísticas que ocurren en o cerca del nervio CPE en la rodilla incluye los quistes intraneurales del nervio CPE, los quistes extraneurales de la articulación tibioperonea superior, el sarcoma sinovial, el mixoma intramuscular y tumores que presenten degeneración quística (schwannoma, neurofibroma) $)^{3,9}$.

El abordaje de estas lesiones actualmente recomendado, basado en la teoría articular, consiste en la descompresión del quiste y la ligadura del pedículo del mismo (la rama articular del CPE) $)^{3,8,10}$. Aunque la rama articular es esencial en el desarrollo de estos quistes, la articulación tibioperonea superior es el lugar primario del que surge esta entidad. Por tanto, la resección de la articulación tibioperonea superior patológica y su membrana sinovial es una parte necesaria del procedimiento en determinados $\operatorname{casos}^{12,15}$.

En el estudio clínico con mayor casuística publicado al respecto, la descompresión del quiste y ligadura de la rama articular elimina la recurrencia sin la necesidad de la resección de la pared del quiste ${ }^{10,12,18}$.

Este enfoque simplifica la operación evitando la disección intraneural y la resección del quiste, minimizando los riesgos asociados (déficit neurológico y dolor neuropático) ${ }^{10,12}$.

A menudo, la descompresión del quiste produce una mejoría clínica a los pocos días de la cirugía, la cual continúa de forma progresiva y constante durante los meses siguientes. Incluso en los casos de recurrencia del quiste intraneural, una nueva intervención pueden lograr excelentes resultados. Un análisis de los resultados en la literatura revisada mostró que los mejores resultados logrados fueron en relación con el control del dolor. La recuperación motora es menos predecible y variable. Dado que el nervio CPE en general no se recupera tan fácilmente como otros nervios, determinar la evolución es más difícil ${ }^{11,12}$. Aunque los resultados en cuanto a la recuperación motora son más pobres que respecto al alivio del dolor, hay algunos pacientes que pueden presentar una marcada mejoría tras la cirugía.

\section{Quistes intraneurales en la edad pediátrica}

Las neuropatías del nervio CPE en niños son raras, y por lo general relacionadas con diversas lesiones por compresión o trauma directo del nervio. Las neuropatías compresivas del nervio CPE en edad pediátrica pueden ser debidas a una compresión postural por cruzar las piernas, pérdida de peso y anorexia, por compresión intrauterina o por trauma en el parto. La neuropatía por atrapamiento es un mecanismo de lesión menos frecuente en la edad pediátrica. El nervio CPE puede quedar atrapado en la rodilla por una exostosis ósea, por el tendón del músculo peroneo lateral largo, o por diversas lesiones tales como schwannomas o quistes intraneurales ${ }^{8}$.

Los quistes intraneurales del nervio CPE son extremadamente raros en la población pediátrica. En nuestra revisión de la literatura en Inglés hemos encontrado 4 casos publicados de quistes intraneurales en población pediátrica. El primero, por Nicholson et al. fue publicado en 1995, Johnston et al. y Aprin et al. publicaron sus casos casi simultáneamente en 2007, y el último, de Frank et al. se publicó en el año 2008. Estos artículos no incluyen en su revisión otros dos casos publicados en la literatura, en sendos artículos en los que se hace hincapié en la utilidad de la ecografía en el diagnóstico en el primer caso $^{4}$, y en 
Tabla 1

Síntesis de las características de los pacientes pediátricos revisados con quistes intraneurales del nervio CPE

\begin{tabular}{|c|c|c|c|c|c|c|c|c|}
\hline & Nicholson & Aprin & Johnston & Scherman & Leijten & Frank & Robla \#1 & Robla \#2 \\
\hline Edad (años) & 4 & 4 & 4 & 9 & 12 & 12 & 6 & 9 \\
\hline $\begin{array}{l}\text { Tiempo de } \\
\text { evolución } \\
\text { de la clínica }\end{array}$ & $\begin{array}{c}2 \\
\text { meses }\end{array}$ & $\begin{array}{c}10 \\
\text { meses }\end{array}$ & $\begin{array}{l}\text { Varios } \\
\text { meses }\end{array}$ & $\begin{array}{c}6 \\
\text { meses }\end{array}$ & $\begin{array}{c}2 \\
\text { semanas }\end{array}$ & $\begin{array}{c}1 \\
\text { mes }\end{array}$ & $\begin{array}{c}4 \\
\text { meses }\end{array}$ & $\begin{array}{c}3 \\
\text { meses }\end{array}$ \\
\hline $\begin{array}{l}\text { Exámen } \\
\text { físico }\end{array}$ & $\begin{array}{l}\text { Pié } \\
\text { caído }\end{array}$ & $\begin{array}{l}\text { Pié } \\
\text { caído }\end{array}$ & $\begin{array}{l}\text { Pie } \\
\text { caído }\end{array}$ & $\begin{array}{l}\text { Pie } \\
\text { caído }\end{array}$ & $\begin{array}{l}\text { Pie } \\
\text { caído }\end{array}$ & $\begin{array}{l}\text { Pie } \\
\text { caído }\end{array}$ & $\begin{array}{l}\text { Pie } \\
\text { caído }\end{array}$ & $\begin{array}{l}\text { Pie } \\
\text { caído }\end{array}$ \\
\hline $\begin{array}{l}\text { Tumoración } \\
\text { palpable }\end{array}$ & $\mathrm{Si}$ & $\mathrm{Si}$ & No & No & $\mathrm{Si}$ & No & $\mathrm{Si}$ & $\mathrm{Si}$ \\
\hline $\begin{array}{l}\text { Trauma } \\
\text { previo }\end{array}$ & No & No & No & $\mathrm{Si}$ & No & $\mathrm{Si}$ & No & No \\
\hline $\begin{array}{l}\text { Tiempo de } \\
\text { evolución hasta } \\
\text { la recuperación } \\
\text { motora }\end{array}$ & $\begin{array}{c}4 \\
\text { meses }\end{array}$ & $\begin{array}{c}4 \\
\text { meses }\end{array}$ & $\begin{array}{c}12 \\
\text { meses }\end{array}$ & - & - & $\begin{array}{c}18 \\
\text { meses }\end{array}$ & $\begin{array}{c}12 \\
\text { meses }\end{array}$ & $\begin{array}{c}12 \\
\text { meses }\end{array}$ \\
\hline $\begin{array}{l}\text { Grado de } \\
\text { recuperación }\end{array}$ & Completa & Completa & Completa & Desconocido & Desconocido & Completa & Completa & Completa \\
\hline
\end{tabular}

las características histopatológicas en el segundo9.

La mayor serie de casos existente, publicada por Spinner et al., no menciona específicamente la existencia de casos en niños, pero describe que el rango de edad de sus 24 casos está entre 12 y 68 años ${ }^{11}$. De la misma manera, Leijten et al. en el año 1992 cita un rango de edad de 9 a 74 años en su revisión de la literatura ${ }^{4}$.

De estos 6 casos pediátricos que encontramos en nuestra revisión (Tabla 1), el rango de edad fue de 4 a 12 años; 3 casos tenían 4 años de edad en el momento del diagnóstico, otro caso 9 y los dos últimos casos 12 años. Todos los casos tuvieron una historia de debilidad motora progresiva hasta que presentaron un pie caído, con un rango de tiempo de evolución del déficit de 2 semanas a varios meses. Al igual que los casos de quistes intraneurales en adultos, en estos 6 casos revisados, los síntomas sensitivos y el dolor referido fueron variables. Sólo dos de ellos presentaban un antecedente previo de traumatismo en la rodilla, y 3 de ellos tenían una tumoración palpable a nivel del cuello del peroné. La edad temprana de presentación sugiere una posible laxitud capsular o un defecto en la misma en estos pacientes.

Todos los pacientes fueron operados tras el diagnóstico de quiste intraneural. En tres de los casos intraoperatoriamente se encontró una conexión entre la rama articular y la articulación tibioperonea superior, y sólo en dos de los 6 casos revisados se ligó y se dividió la rama articular, desconectándola de la articulación. La evolución postoperatoria en los pacientes en los que se mencionó su seguimiento fue excelente, con una recuperación completa desde el punto de vista motor. El tiempo de evolución postoperatorio hasta la recuperación completa fue de 9,5 meses.

Los dos nuevos casos publicados en este artículo comparten algunas de las características de los casos pediátricos publicados previamente. Estos 2 pacientes presentaron una historia de varios meses de evolución de dolor y déficit motor distal en la pierna afecta (presentando una marcha en stepagge), pero sin déficit sensitivo. Ambos pacientes fueron operados según los principios de la teoría articular publicada por Spinner, ligando y seccionando la rama articular y descomprimiendo el quiste, demostrándose una vez más que es una técnica que ofrece una solución permanente, sin presentar recidiva del quiste, y con un mínimo riesgo para el nervio afectado. Ambos pacientes se recuperaron de su déficit motor por 
Tabla 2

Comparación entre los datos recogidos de una amplia serie de casos en adultos y los casos pediátricos publicados

\begin{tabular}{|l|c|c|}
\hline & $\begin{array}{c}\text { Casos en edad adulta } \\
\text { (Spinner et al.) (24 casos) }\end{array}$ & $\begin{array}{c}\text { Casos pediátricos } \\
\text { ( } 8 \text { casos) }\end{array}$ \\
\hline Sexo (Hombres:Mujeres) & $3,8: 1$ & $3: 1$ \\
\hline Edad media (años) & 43 & 3,2 \\
\hline Tiempo medio de progression de la clínica (meses) & $83 \%$ & $100 \%$ \\
\hline Déficit para la dorsiflexión del pié en el momento del diagnóstico & 2,5 & 0,5 \\
\hline Grado MRC (media) en el momento del diagnóstico & 3,3 & 4,6 \\
\hline Grado MRC (media) al final del seguimiento & $58 \%$ & $75 \%$ \\
\hline Déficit para la eversión del pié en el momento del diagnóstico & 3,5 & 1,2 \\
\hline Grado MRC (media) en el momento del diagnóstico & 4,2 & 4,5 \\
\hline Grado MRC (media) al final del seguimiento & $62 \%$ & $62 \%$ \\
\hline Síntomas sensitivos & $58 \%$ & $25 \%$ \\
\hline Trauma previo & $67 \%$ & $62 \%$ \\
\hline Tumoración palpable & $83 \%$ & $30 \%$ \\
\hline Signo de Tinel & & $62 \%$ \\
\hline
\end{tabular}

completo meses después de la descompresión del quiste. Este hecho coincide con el resto de los casos pediátricos existentes en la literatura, los cuales también presentaron una recuperación motora ad integrum, a diferencia de los casos de quistes intraneurales en adultos, comentados previamente. La Tabla 2 muestra una comparación entre los casos pediátricos y los casos en población adulta en relación con esta cuestión.

\section{Conclusión}

Aunque los quistes intraneurales del nervio CPE son poco frecuentes en los adultos, ha sido posible describir su etiopatogenia y sus características clínicas y de imagen. En la población pediátrica, estos quistes intraneurales son extremadamente raros, y sólo se han publicado unos pocos casos. Las características clínicas de estas lesiones en los niños parecen ser similares a las de los adultos, excepto en relación con el grado de recuperación del déficit motor. A diferencia de la recuperación motora incompleta observada en la población adulta tras la descompresión de los quistes intraneurales, los casos pediátricos publicados, incluyendo nuestros dos nuevos casos, muestran una excelente respuesta a la descompresión presentando una recuperación casi completa de la función motora. Los quistes intraneurales deben ser considerados en el diagnóstico diferencial de niños que presenten deformidades del pie de nueva aparición, una marcha en stepagge, o una parálisis del nervio ciático poplíteo externo, especialmente si se trata de una afectación aislada de su rama profunda.

\section{Agradecimiento}

Los autores desean agradecer al Dr Robert Spinner, de la Clínica Mayo de Rochester, Minnesota, Estados Unidos, por el valioso consejo aportado y la revisión del manuscrito final. 


\section{Bibliografía}

1) Aprin, H., Weinberg, J., Lustrin, E.S., Abrutyn, D.: Peroneal nerve palsy due to an intraneural ganglion: a case report of a 4 1/2-year-old boy. Am J Orthop. 2007 Mar; 36: E40-42.

2. Elangovan, S.E., Odegard, G.M., Morrow, D.A., Wang, H., Hébert-Blouin, M.N., Spinner, R.J.: Intraneural ganglia: a clinical problem deserving a mechanistic explanation and model. Neurosurg Focus. 2009 Feb; 26: E11.

3. Johnston, J.A., Lyne, D.E.: Intraneural ganglion cyst of the peroneal nerve in a four-year-old girl: a case report. $\mathrm{J}$ Pediatr Orthop. 2007 Dec; 27: 944-946.

4. Leijten, F.S., Arts, W.F., Puylaert, J.B.: Ultrasound diagnosis of an intraneural ganglion cyst of the peroneal nerve. Case Report. J Neurosurg. 1992 Mar; 76: 538-540.

5. Lowenstein, J., Towers, J., Tomaino, M.M.: Intraneural ganglion of the peroneal nerve: importance of a timely diagnosis. Am J Orthop. 2001; 30: 816-819.

6. McCarthy, C.L., McNally, E.G.: The MRI appearance of cystic lesions around the knee. Skeletal Radiol. 2004 Apr; 33: 187-209.

7. Nicholson, T.R., Cohen, R.C., Gratten-Smith, P.J.: Intraneural ganglion of the common peroneal nerve in a 4year-old boy. J Child Neurol.1995; 10: 213-225.

8. Nucci, F., Artico, M., Santor, A.: Intraneural synovial cyst of the peroneal nerve: report of two cases and review of the literature. Neurosurgery. 1990; 26: 339-343.

9. Scherman, B.M., Bilbao, J.M., Hudson, A.R., Briggs, S.J.: Intraneural ganglion: a case report with electron microscopic observations. Neurosurgery. 1981 Apr; 8: 487-490.

10. Spinner, R.J., Amrami, K.K.: What's new in the management of benign peripheral nerve lesions? Neurosurg Clin $\mathrm{N}$ Am. 2008 Oct; 19: 517-531.

11. Spinner, R.J., Atkinson, J.L., Scheithauer, B.W., et al.: Peroneal intraneural ganglia: the importance of the articular branch. Clinical series. J Neurosurg. 2003 Aug; 99: 319-329.
12. Spinner, R.J., Atkinson, J.L.D., Scheithauer, B.W.: Peroneal intraneural ganglia: the importance of the articular branch. A unifying theory. J Neurosurg. 2003 Aug; 99: 330333.

13. Spinner, R.J., Carmichael, S.W., Wang, H., Parisi, T.J., Skinner, J.A., Amrami, K.K.: Patterns of intraneural ganglion cyst descent. Clin Anat. 2008 Apr; 21: 233-245.

14. Spinner, R.J., Desy, N.M., Rock, M.G., Amrami, K.K.: Peroneal intraneural ganglia. Part I. Techniques for successful diagnosis and treatment. Neurosurg Focus. 2007 Jun 15; 22: E16.

15. Spinner, R.J., Desy, N.M., Rock, M.G., Amrami, K.K.: Peroneal intraneural ganglia: part II: lessons learned and pitfalls to avoid for successful diagnosis and treatment. Neurosurg Focus 2007; 22: E27.

16. Spinner, R.J., Scheithauer, B.W., Amrami, K.K.: The unifying articular (synovial) origin of intraneural ganglia: evolution-revelation-revolution. Neurosurgery. 2009 Oct; 65: 115-124.

17. Spinner, R.J., Wang, H., Carmichael, S.W., Amrami, K.K., Scheithauer, B.W.: Epineurial compartments and their role in intraneural ganglion cyst propagation: an experimental study. Clin Anat. 2007 Oct; 20: 826-833.

18. Young, N.P., Sorenson, E.J., Spinner, R.J., Daube, J.R.: Clinical and electrodiagnostic correlates of peroneal intraneural ganglia. Neurology. 2009 Feb 3; 72: 447-452.

Robla-Costales, J.; Socolovsky, M.; Dubrovsky, A.; Astorino, F.; Fernández-Fernández, J.; Ibáñez-Plágaro, J.; García-Cosamalón, J.: Quistes intraneurales del nervio ciático popliteo externo en edad pediátrica: presentación de 2 casos y revisión de la literatura. Neurocirugía 2011; 22: 324-331.

Correspondencia: Javier Robla Costales. Servicio de Neurocirugía. Hospital de León. C/ Altos de Nava s/n, 24701 León. España. 